Check for updates

Cite this: Mater. Chem. Front., 2019, 3, 12

Received 5th August 2018, Accepted 30th October 2018

DOI: $10.1039 / c 8 q m 00383 a$

rsc.li/frontiers-materials

\section{AlEgen bioconjugates for specific detection of disease-related protein biomarkers}

\author{
Kok Chan Chong, Fang Hu (D) and Bin Liu (D) *
}

\begin{abstract}
Mis-regulation of certain proteins and enzymes is often identified as an indicator of many pathologies, and these disease-related proteins can be used as biomarkers to diagnose disease onset and progression. Indeed, bioprobes with various working mechanisms have been developed for the sensing of these protein biomarkers. Particularly, fluorophore-based probes have favorably been welcomed due to their outstanding properties such as easy synthesis, facile modification, real-time, on-site responsiveness, and noninvasiveness. However, conventional fluorophores usually suffer from aggregationcaused quenching, which greatly affects their sensitivity and photostability. To overcome this challenge, fluorogens with aggregation-induced emission features have been developed, which offer higher signal to noise ratios and better resistance to photobleaching in sensing and imaging. In this review, the recent development of specific light-up bioprobes based on AlEgen bioconjugates for sensing and imaging of disease-related protein biomarkers is summarized and discussed with the aim to serve as a guidance for the design of state-of-the-art AlEgen probes for a wide range of biomedical applications.
\end{abstract}

\section{Introduction}

Currently, fluorophores are broadly employed for a vast array of applications ranging from photovoltaics and colour displays to optical imaging. However, although they are widely welcomed as one of the most promising tools for real-time, on-site responsive, non-invasive bio-imaging and diagnostic sensing with high temporal-spatial resolution, they have several shortcomings, which have limited their use in real-life applications. Due to the notorious aggregation-caused quenching (ACQ) effect, the fluorescence of most fluorophores is reduced greatly when they are used in high concentration, which compromises the sensitivity of bio-imaging. ${ }^{1}$ For example, fluorescein, a conventional fluorophore, emits strong green fluorescence as an isolated molecule in dilute solution (Scheme 1a). However, once fluorescein molecules aggregate, they experience strong intermolecular $\pi-\pi$ stacking interactions, and the emission of these aggregates is mostly quenched via nonradiative pathways. Therefore, these fluorophores can only be employed at low concentrations, which result in poor photostability. In addition, without introducing tedious separation and washing processes to remove unbound fluorophores, the inherent fluorescence of conventional fluorophores can result in high background signals, which in turn result in a low signal-to-noise ratio (SNR) during the visualization of biological processes.

Since the discovery of fluorogens with aggregation-induced emission features (AIEgens), AIEgens have successfully attracted

Department of Chemical \& Biomolecular Engineering, National University of Singapore, 117585, Singapore. E-mail: cheliub@nus.edu.sg

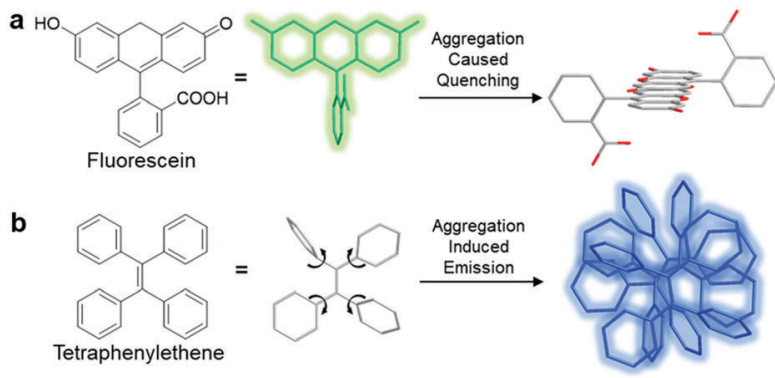

Scheme 1 Schematic illustration of the ACQ and AIE phenomena using (a) fluorescein and (b) tetraphenylethene, respectively, as typical examples.

the attention of the scientific community owing to their highly desirable characteristics. ${ }^{2}$ In contrary to common fluorophores, AIEgens are faintly emissive in the molecular state, where the attached phenyl rings can rotate freely and hence exhaust the excitation energy in the process through a non-radiative decay pathway. Upon the formation of aggregates, the restriction of intramolecular motions (RIM) plays a critical role in blocking non-radiative decay pathways by hindering the rotation and vibration of AIEgens, leading to a highly emissive state (Scheme 1b). Moreover, AIEgens can hardly form any intermolecular $\pi-\pi$ stacking interactions during aggregation due to their non-planar molecular structures, and thus they do not suffer from the ACQ effect. Benefiting from these properties, well-designed AIEgens can provide better SNR in bio-imaging, and a higher operative concentration is allowed for the enhancement of photostability. 
Considering these advantages, light-up probes based on AIEgens can be engineered and constructed to show nearly no emission in aqueous media, which turn on their fluorescence upon interaction with target analytes. Among the various target analytes, proteins, a type of biomacromolecule that performs a spectrum of functions in the body, including catalysis of metabolism, transport of molecules across cells and even cell apoptosis, have become the focus of biological studies. It is a well-established fact that the abnormal expression of specific proteins and enzymes is usually associated with certain pathogenies such as Alzheimer's disease and cancer. ${ }^{3-5}$ Thus, these proteins can undoubtedly be employed as biomarkers for the diagnosis of health disorders. Decades of pharmaceutical research have pointed towards one conclusion: the earlier a disease is detected, the greater the possibility it can be alleviated and treated. However, these protein biomarkers exist only at the molecular level during the early stage of diseases, which makes the early stage pathology study a great challenge. Thus, there is a need to develop ultrasensitive diagnostic tools that can identify diseaserelated proteins in biomarker levels before advanced clinical signs and symptoms manifest. AIE bioprobes have distinct advantages including high SNR light-up property with reduced false-positive response, and thus are promising to solve this dilemma.

The charged AIEgens reported in the earlier works rely on electrostatic and hydrophobic interactions with analytes and displayed less than desirable responses towards analytes of interest in biological study due to the presence of interfering species in complex media. ${ }^{6}$ Hence, the inclusion of a recognition element that has high affinity with the target protein is crucial to significantly enhance the selectivity of these bioprobes. In this regard, biomolecules with highly specific molecular recognition towards protein biomarkers, such as peptides and carbohydrates, can be applied and incorporated as a targeting moiety for specific AIEgen light-up bioprobes. In fact, a variety of AIEgen bioconjugate probes demonstrating thrilling results have been designed throughout the years. ${ }^{7-11}$

Distinct from comprehensive reviews on AIE reported in the literature, ${ }^{12-20}$ this mini-review focuses on the recent development of specific light-up bioprobes based on AIEgen bioconjugates for disease-related protein sensing. The design rationale, operation mechanism of bioconjugated AIEgen probes and commonly used conjugation techniques are firstly introduced. In the following sections, representative examples of AIEgen bioconjugates for protein biomarker detection are discussed. Finally, the future perspective of AIEgen bioconjugates for protein sensing is discussed to motivate the design and development of novel bioconjugated AIE-based probes for biomedical applications.

\section{Probe design principle}

The family of AIEgens has rapidly expanded throughout the years of research and effort, and one common feature is shared among most AIEgens: they have phenyl ring-based propellers, which can dissipate excitation energy efficiently via free motions. Once such AIEgen aggregate, RIM sets in to activate the radiative decay pathway, inducing fluorescence "turn-on". Among the AIEgens, highly tunable tetraphenylethene (TPE) derivatives are popular choices as the fluorescent core of AIE bioprobes, with variants of TPE displaying different emission colors. Tetraphenylsilole (TPS), an AIEgen derived from hexaphenylsilole has also been favored in the design of bioconjugated AIE probes. Other AIEgens that show synergistic phenomena, such as salicylaldehyde azine (SA) with AIE and excited-state intramolecular proton transfer (ESIPT) characteristics, are also desirable for the construction of remarkably sensitive AIE bioprobes. $^{21}$ The AIEgens used for the construction of bioconjugated probes are summarized in Table 1.

By considering the RIM mechanism, which is responsible for the fluorescence turn-on of AIEgen, it is of high priority to ensure that AIEgen probes have good water-solubility and hence remain non-emissive in the molecular state in aqueous media until RIM activation triggered by the analyte of interest. Encouragingly, most biomolecules have good water-solubility, and the direct ligation of biomolecules with hydrophobic AIEgens can form hydrophilic AIEgen bioprobes with low fluorescent background. Although few biomolecules are sufficiently hydrophilic to bring conjugated AIEgens into aqueous media, the introduction of hydrophilic linkers such as polyethylene glycol (PEG) or short hydrophilic peptides, for example, in between AIEgen and recognition biomolecules, can substantially improve the water-solubility of AIEgen bioconjugate probes. The selection of biomolecules for the construction of probes generally follows two operation mechanisms, as shown in Scheme 2. The first is the introduction of biomolecules that can serve as ligands to recognize target proteins (Scheme 2a). Upon biomoleculeprotein recognition interaction, the intramolecular motions of the probes are inhibited, and the fluorescence is turned on for protein detection. The other is the introduction of hydrophilic biomolecules that can be cleaved by the target enzyme (Scheme 2b). After cleavage of the hydrophilic moieties, the remaining hydrophobic AIEgens form aggregates and exhibit RIM-induced fluorescent signals to achieve enzyme detection.

Integration of complex-structured biomolecules into AIEgens is achievable through simple yet elegant bioconjugation techniques. A summary of the commonly used coupling chemistry to yield AIEgen-based bioprobes is listed in Scheme 3. The first two methods involve the direct conjugation of primary amine groups with activated carboxyl or isothiocyanate (NCS) groups to create stable amide (Scheme 3a) and thiourea linkages (Scheme 3b), respectively, between the AIEgen and the recognition element. Although this is a facile method, one should take into consideration that due to the abundance of amine and carboxylic groups in biomolecules such as peptides, this nonchemoselective reaction may result in unwanted side reactions that hinder the selectivity of bioprobes. Another commonly used conjugation strategy is click chemistry, where a pair of clickable chemical groups are introduced into AIEgens and biomolecules for convenient conjugation. Among the various click reactions, copper(I)-catalysed azide-alkyne cycloaddition (Scheme 3c) and thiol-maleimide Michael addition (Scheme 3d) are the two most popular methods for bioconjugation between AIEgens and biomolecules since they proceed rapidly and efficiently with minimal by-products. 
Table 1 AlEgens used for the construction of bioconjugated probes

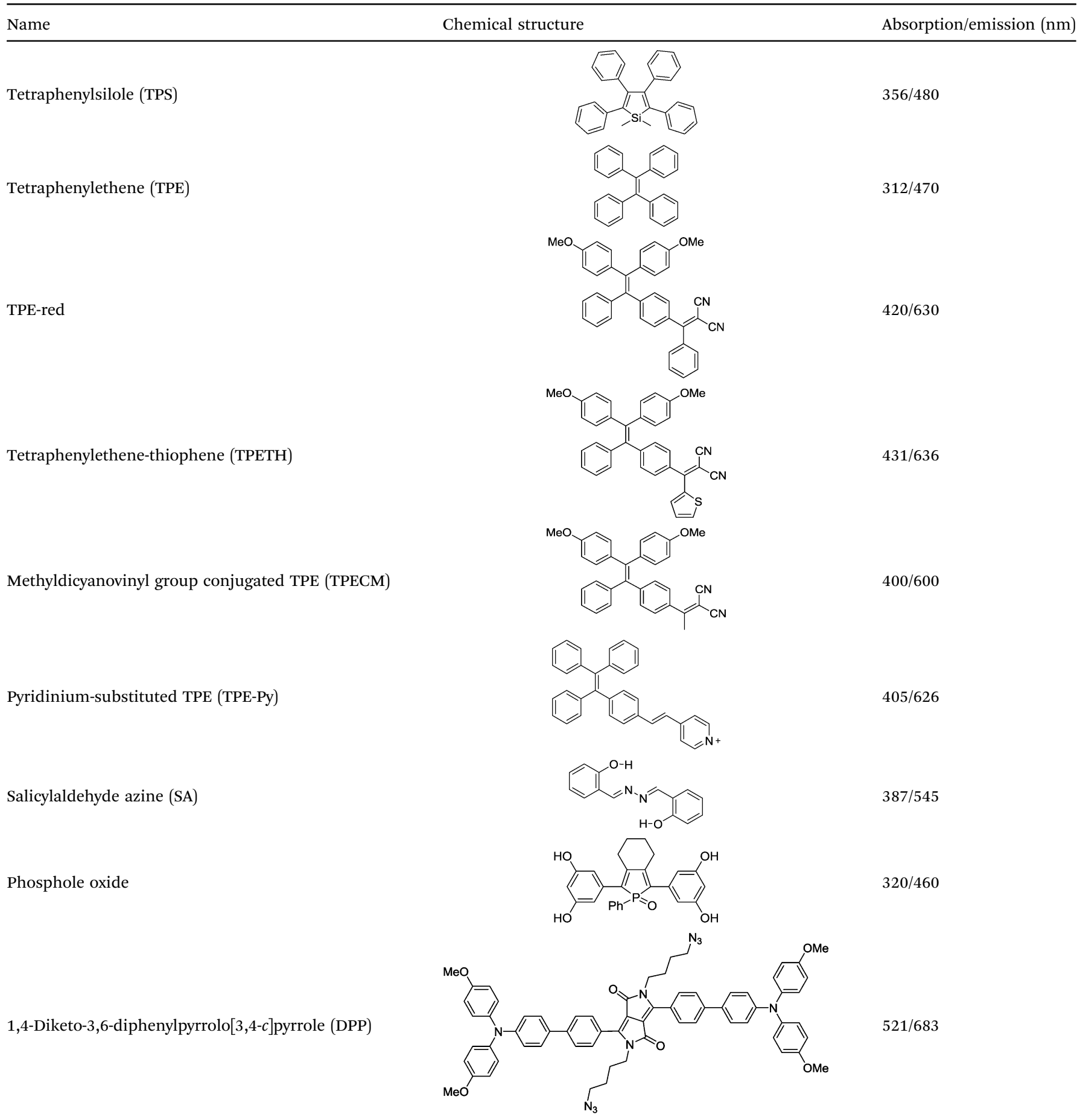

\section{Applications of AlEgen bioconjugate in disease-related protein detection}

Following the design principles and bioconjugation techniques, numerous AIEgen bioconjugate probes have been invented and explored for disease-related protein detection. In this section, AIEgen bioconjugate-based protein detection is classified into two categories based on the operation mechanisms of the AIEgen bioprobe. After the discussion on protein detection, advanced
AIEgen bioconjugates with multi-functions for detection and therapy in one system are demonstrated in detail.

\subsection{AIEgen bioconjugate probes based on protein-binding and recognition}

Based on the knowledge of specific binding between host proteins and target guest molecules, researchers have developed a variety of AIEgen bioprobes for specific protein binding and sensing with 
a
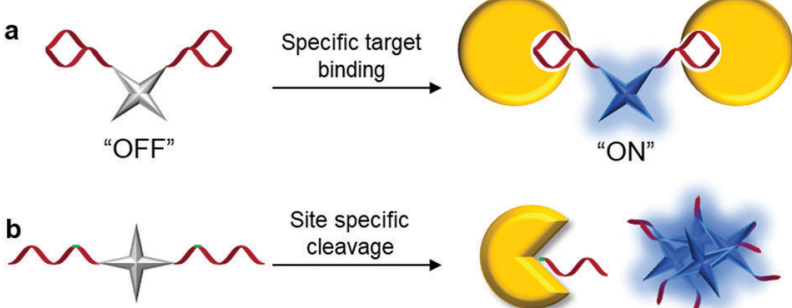

"OFF"

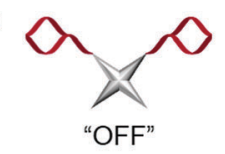

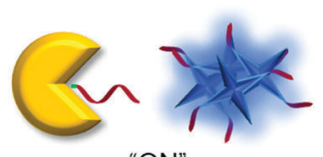

"ON"
Scheme 2 Typical operation mechanisms for AlEgen bioconjugate-based probes.

$$
\text { b } \mathrm{R}_{1}-\mathrm{N}=\mathrm{C}=\mathrm{S}+\mathrm{R}_{2}-\mathrm{NH}_{2} \longrightarrow \mathrm{R}_{2}-\mathrm{NH}_{2} \stackrel{\mathrm{EDC}}{\mathrm{NHS}}
$$

Scheme 3 Common coupling reactions between functionalized AIEgens and recognition units.

various biomolecules conjugated as the targeting moiety, ranging from peptides to carbohydrates.

3.1.1 AIEgen-peptide bioconjugate. Peptides exist as sequences of amino acid residues, which assume a variety of shapes forming from helices, sheets and loops, or a combination of all. They have extensively been applied as recognition ligands in the design of bioimaging probes due to their appropriate size, easy synthesis and functionalization, and high affinity and specificity with targeted biomarkers. Thus far, AIEgen-peptide bioconjugates for diseaserelated protein detection have been most highly developed.

In 2012, Liu and Tang et al. pioneered the first design of an AIEgen-peptide bioconjugate, TPS-2cRGD (Fig. 1a), for the light-up detection of the cancer-related protein, integrin $\alpha_{v} \beta_{3}{ }^{7}$ This cyclic-RGD (cRGD) peptide-conjugated TPS probe was devised with click chemistry to specifically target integrin $\alpha_{v} \beta_{3}$, a crucial protein biomarker existing in several different cancer types. Upon specific binding to integrin, the green emission from TPS-2cRGD can be quickly turned on as a result of the RIM mechanism, as illustrated in the scheme in Fig. 1b. This allows quantitative detection of integrin by the probe in solution with high sensitivity, as shown by the 182 -fold brighter fluorescence in the presence of integrin $\alpha_{v} \beta_{3}$. Furthermore, this bioprobe is able to distinguish integrin-overexpressed HT-29 cells from MCF-7 cells with a low level of integrin in cell imaging, proving its ability to identify integrin-positive cells over integrin-negative ones (Fig. 1c). Overall, their work represented the first AIEgen bioconjugate for specific biomarker detection and imaging, which opened a new avenue for thorough research into peptide-conjugated AIE biosensing probes.

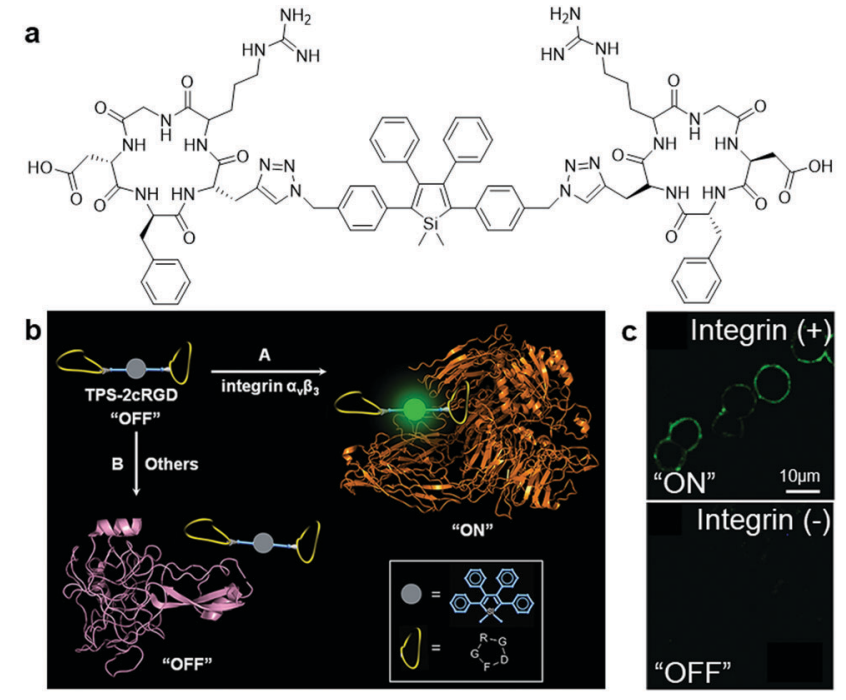

Fig. 1 (a) Chemical structure of TPS-2cRGD, (b) schematic illustration of the operation mechanism of TPS-2CRGD for integrin $\alpha_{v} \beta_{3}$ binding and imaging, and (c) confocal fluorescent images of integrin-positive (top) and integrin-negative cells (bottom) after incubation with TPS-2CRGD $(2 \mu \mathrm{M})$. Adapted and modified with permission from ref. 7. Copyright 2012 American Chemical Society.

Based on the same mechanism, Zhang, and Zhao et al. designed a dual-responsive bioconjugate probe based on a TPE derivative, TPE-AP2H. ${ }^{22}$ With the attached IHGHHIISVG (AP2H) peptide ligand, TPE-AP2H can respond specifically to the membrane-anchored lysosomal protein transmembrane 4 beta (LAPTM4B), which is overexpressed in many solid tumours. Meanwhile, the proton-responsive histidine residue in AP2H can reinforce the stronger binding of TPE-AP2H with the LAPTM4B protein under the acidic extracellular environment of tumour cells, thus enhancing RIM and leading to an increase in the fluorescence intensity of TPE-AP2H. Benefiting from the dual-responsive process of recognition and acidic environment, this bioprobe was successfully employed to monitor the LAPTM4B protein specifically with a high SNR.

Guided by the prior works, several AIEgen-peptide bioconjugates were reported successively for specific protein detection. ${ }^{23,24}$ However, the absorption and emission spectra of TPE and TPS are located in the ultraviolet and blue region, which is not only harmful to biosystems, but may affect the probe sensitivity due to the low penetrating depth of the excitation light and emission overlapping with induced cell autofluorescence. Thus, to further improve the performance of probes, visible light-excited and red-emissive AIEgens were developed and introduced for the design of AIEgen-peptide bioconjugates. One of the successful examples was explored by Zhang et al., where TPE was successfully modified with electron-donating alkoxy groups and electron-withdrawing dicyano group to formulate TPE-red. The donor-acceptor structure enabled TPE-red to exhibit red-shifted absorption and emission peaks at 420 and $630 \mathrm{~nm}$, respectively. By the conjugation of TPE-red with the targeting peptide AP2H, the obtained TPE-red-2AP2H (Scheme 4) realized intracellular detection of the LAPTM4B protein 


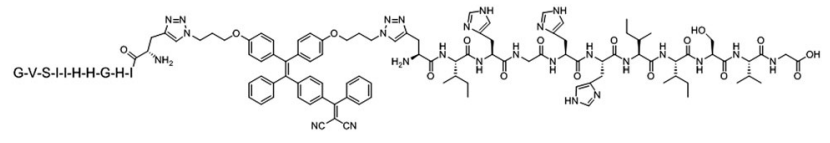

Scheme 4 Chemical structure of TPE-red-AP2H.

with an SNR as high as $930 .^{25}$ Another red-emissive tetraphenylethene-thiophene (TPETH)-based AIEgen bioconjugate with the HAIYPRH (T7) peptide at two ends, TPETH-2T7, was proposed by Liu et al. to target and detect transferrin receptor (TfR), a transmembrane glycoprotein that is found to be upregulated in highly proliferative cancer cells. ${ }^{26}$ The as-developed probe was almost non-luminescent in aqueous solution and emitted turn-on red fluorescence only in the presence of TfR, achieving an emission intensity increase of nearly 90 -fold. On a side note, a similar bioprobe, TPE-2T7, was fabricated recently for the simple, yet versatile detection of serum transferrin receptor (sTfR) in urine samples with a detection limit of $0.27 \mu \mathrm{g} \mathrm{mL} \mathrm{m}^{-1}$, which is lower than the sTfR level in patients suffering from iron deficiency anaemia, thus exhibiting the potential of AIEgen bioconjugates for clinical application. ${ }^{27}$

Other peptide sequences showing synergistic functions, such as one that aid in promoting RIM, can also be incorporated into AIE bioprobes for increased sensitivity in target detection. One of the examples is TPE-Py-FFGYSA (Fig. 2a), an AIEgen-peptide bioconjugate combining the pyridiniumsubstituted TPE salt (TPE-Py) with YSAYPDSVPMMS peptide (YSA), which targets the cancer cell overexpressed transmembrane receptor tyrosine kinase EphA2 and self-assembled aided peptide unit FFG. This bioprobe was synthesized for EphA2 targeted cancer cell imaging with enhanced fluorescence output. ${ }^{28}$ The simple incorporation of FFG between TPE-Py and YSA can enhance the fluorescent signal output of the probe significantly since FFG with aromatic rings favours supramolecular selfassembly, which in turn forms a tighter probe assembly and promotes more effective RIM of TPE-Py (Fig. 2b). Compared to TPE-Py-YSA without the FFG self-assembled aided unit, the fluorescence intensity of TPE-Py-FFGYSA increased by $\sim 3.7$ times

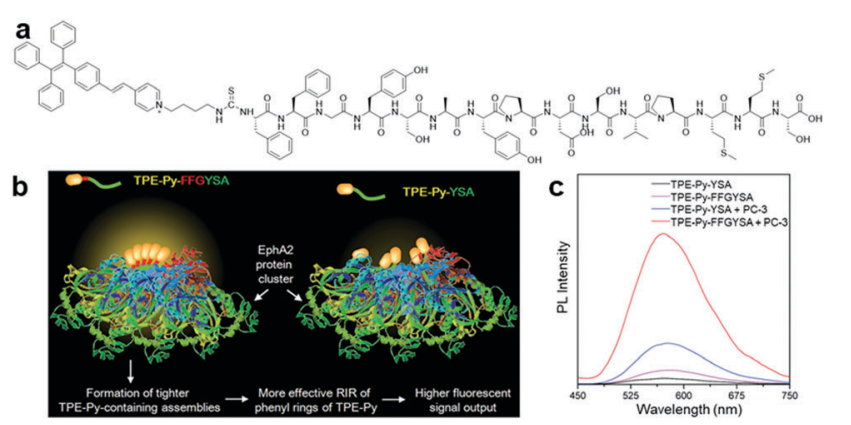

Fig. 2 (a) Chemical structure of TPE-Py-FFGYSA, (b) schematic illustration of the operation mechanism of TPE-Py-FFGYSA for EphA2 sensing with enhanced fluorescent signal output, and (c) PL spectra of TPE-Py-FFGYSA $(1 \mu \mathrm{M})$ and TPE-Py-YSA $(1 \mu \mathrm{M})$ in PBS buffer with and without the addition of EphA2-overexpressed PC-3 cell lysate with excitation at $405 \mathrm{~nm}$. Adapted and modified with permission from ref. 28. Copyright 2017 Royal Society of Chemistry. during the detection of EphA2 in PC-3 cell lysate, as shown in the PL spectra in Fig. 2c. Thus, this new design strategy can potentially aid researchers in creating bioprobes that can detect and image targeted proteins with much higher contrast.

To further increase the specificity of AIEgen peptide bioconjugates, multiple peptide moieties responsive to different target proteins can be integrated into a single combinatorial peptide chain for conjugation with selected AIEgens. A multifunctional peptide conjugated AIEgen (Fig. 3a) was designed by Xia and Lou et al. for long-term nuclear-targeted cancer cell tracking with low toxicity. ${ }^{29}$ This bioprobe consisted of a few parts including TPE-Py, an integrin-targeting peptide RGD, an aminopeptidase $\mathrm{N}$ (CD13)-targeting peptide cNGR, an argininebased cell-penetrating peptide to improve transportation of the probe into cytoplasm, and lastly a nuclear localization signal (NLS) for cell nucleus internalization of the probe (Fig. 3b). As shown in Fig. 3c, after incubating this cNGR-CPP-NLS-RGD-TPE-Py probe (TCNTP) with CD13 and integrin $\alpha_{v} \beta_{3}$-overexpressed A375 cells, the yellow fluorescence of TCNTP was observed to be well overlapped with the blue fluorescence from Hoechst 33258, a nuclear staining dye, which resulted in a high Pearson correlation coefficient of $92 \%$ for 12 hour incubation. On the contrary, when the same experiments were conducted with the negative controls, MDA-MB231 (integrin-positive only) and HT-1080 (CD-13-positive only) cells, there was no noticeable overlapping of fluorescence between TCNTP and Hoechst 33258, indicating its exceptional specific targeting ability. TCNTP can also be utilized for long-term live cell tracing for over 10 days due to the negligible toxicity and strong target binding affinity of the probe.

3.1.2 AIEgen-oligonucleotide bioconjugates. Oligonucleotides are biopolymers composed of negatively charged nucleotides that bear genetic information crucial to all living organisms. Several remarkable AIEgen bioconjugate probes based on the turn-on mechanism upon hybridization between complementary oligonucleotides have been reported for targeted nucleic acid detection. ${ }^{8,30,31}$ Apart from sensing nucleic acids, oligonucleotides can also be utilized for protein recognition. Aptamers, as a group of special, short, single-stranded oligonucleotide molecules, are capable of binding various molecules including protein with high specificity. They are remarkable analogues to antibodies, with several superior features, such as higher stability, cheaper production cost, and easier chemical labelling. Benefiting from these characteristics, the development of aptamer-based biosensing probes has been emphasized in recent years.

An aptamer-modified protein-targeting biosensor based on AIEgen, TPE-aptamer (Fig. 4a), was reported by Shahzad and $\mathrm{Yu}$ et $a .^{32}$ By simply conjugating the immunoglobin heavy mu chain receptor $(\mathrm{IgH} \mu)$-specific aptamer to both ends of the TPE unit, this probe was capable of selectively recognizing IgH $\mu$ overexpressed Burkitt's lymphoma B cells, Ramos B cells, from other cell lines. ${ }^{33}$ TPE-aptamer is weakly fluorescent in blank buffer solution, and lights up only after incubating with Ramos B cells. This is attributed to the RIM process induced by specific binding between the aptamer ligand and $\mathrm{IgH} \mu$ protein on the Ramos B cell surface (Fig. 4b). The probe can detect as little as 100 Ramos B cells without any signal amplification and 


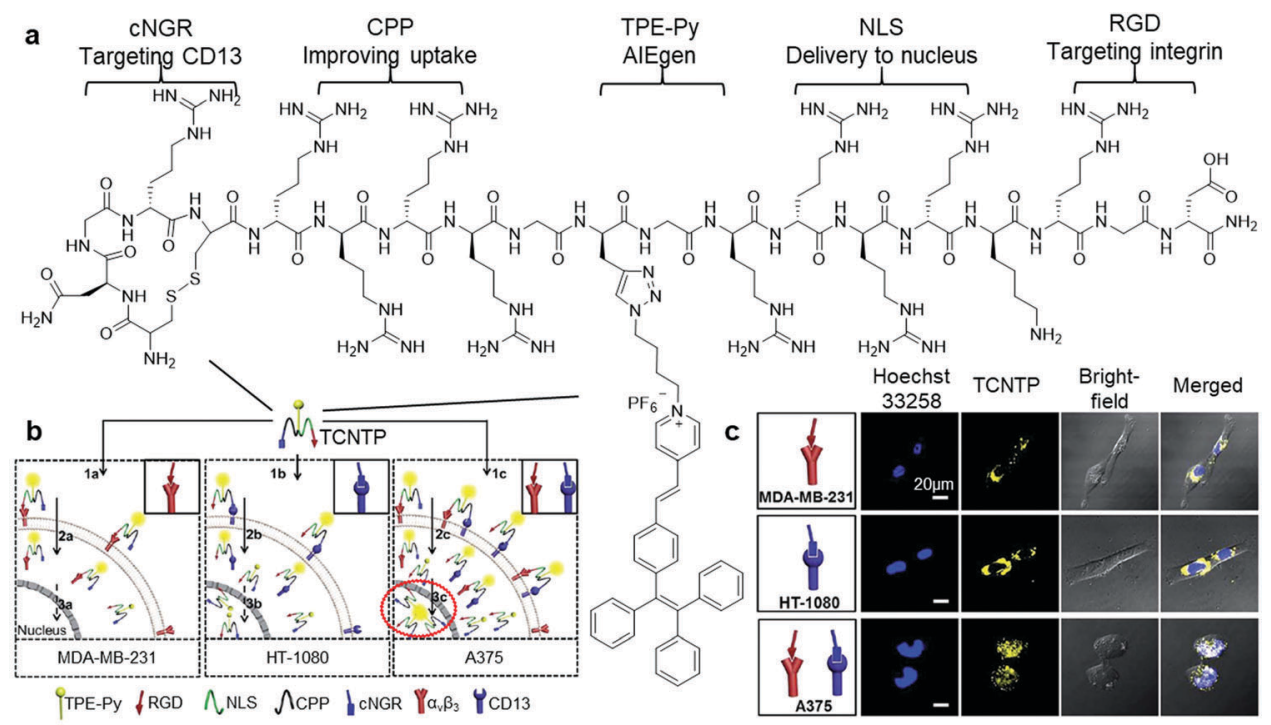

Fig. 3 (a) Chemical structure of TCNTP, (b) schematic illustration of the operation mechanism of TCNTP for nuclear-targeted cancer cell tracking, and (c) confocal fluorescence images of integrin-positive (top), CD13-positive (middle) and both integrin and CD-13 positives cells (bottom) after incubation with TCNTP $(3 \mu \mathrm{M})$. Adapted and modified with permission from ref. 29. Copyright 2017 Royal Society of Chemistry.

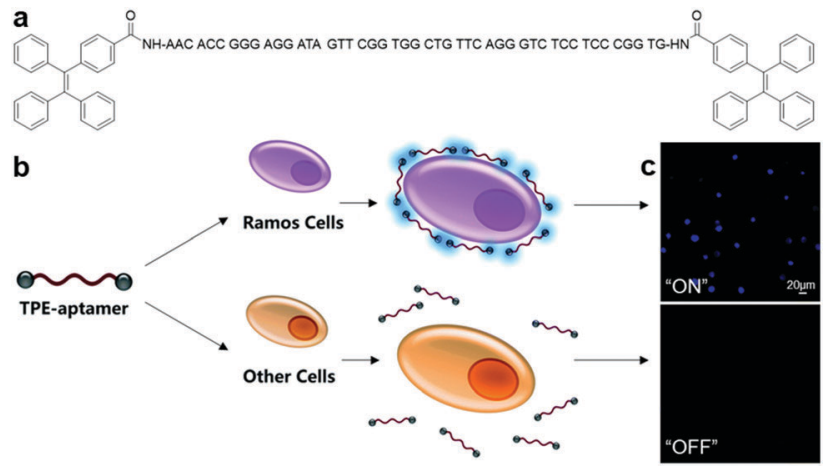

Fig. 4 (a) Chemical structure of TPE-aptamer, (b) schematic of TPEaptamer for Ramos cell-targeted sensing and imaging, and (c) confocal fluorescence images of Ramos cells and other cells incubated with TPE-aptamer $(2.5 \mu \mathrm{M})$. Adapted and modified with permission from ref. 33. Copyright 2017 Royal Society of Chemistry.

displays fluorescence light up with Ramos B cells, which is at least 20-fold higher than that in other cells (Fig. 4c). Aside from adopting the straightforward and fast "mix-and-detect" approach, this assay also performs remarkably in the sensing of Ramos B cells from complicated sample mixtures as well as in specific cancer cell imaging. These advantages allow one to optimistically anticipate the effortless translation of this aptamer-based assay principle for the sensing of other potential targets, for example mutated cells, microorganisms and viruses.

Recently, Liu, Goldys and Tian also devised an TPE-based aptasensor that can a detect trace amount of intracellular interferon-gamma (IFN- $\gamma$ ), a group of cytokines with antipathogenic and anti-cellular proliferative activity. ${ }^{34}$ Cytokines are cell signalling proteins that are closely related with inflammation and disease, and exist in body fluids and tissues at very low concentration up to the picomolar range. With this developed red fluorescent aptasensor, not only the in vitro visualization of IFN- $\gamma$ secretion with a very low detection limit of $2 \mathrm{pg} \mathrm{mL^{-1 }}$ could be achieved, specific localization endogenous IFN- $\gamma$ of $<10 \mathrm{pg} \mathrm{mL} \mathrm{mL}^{-1}$ in real-time was also realized. This facile, yet highly sensitive AIEgen-aptamer bioconjugate may provide a universal detection tool for monitoring ultra-small concentrations of molecules secreted by cells and exploring pathways and dynamic processes of molecules involved in the cellular environment.

3.1.3 AIEgen-carbohydrate bioconjugate. As another type of biomolecules, carbohydrates have also been utilized as a recognition element for protein binding. Also known as saccharides, carbohydrates serve numerous essential roles in living organisms, such as the structural component of other biomolecules and energy storage. Carbohydrate-protein interactions are some of the most crucial events in biological systems as these interactions mediate numerous biological processes, including cell recognition, cell growth, cancer metastasis, inflammation, and infection. Therefore, inspired by these interactions, several highly sensitive and specific bioassays based on AIEgen carbohydrate bioconjugates were developed for the sensing of disease-related proteins.

A group of carbohydrate-protein binding proteins, lectins, has been at the centre of research interest since they are known to take part in interactions with saccharide receptors during initial recognition processes. One of the earliest examples of AIEgen carbohydrate bioconjugates for lectin sensing was cooperatively designed by Sanji and Tanaka et al. ${ }^{9}$ using an AIE-active phosphole oxide and two types of saccharidetargeting moieties, D-mannopyranoside and D-galactopyranosides, which can specifically bind to concanavalin A (Con A) and peanut agglutinin, respectively (Fig. 5a). Upon binding to lectins, probelectin aggregates were formed to turn on bright blue fluorescence (Fig. 5b). Shortly after that, new lectin-targeting TPE-cored 


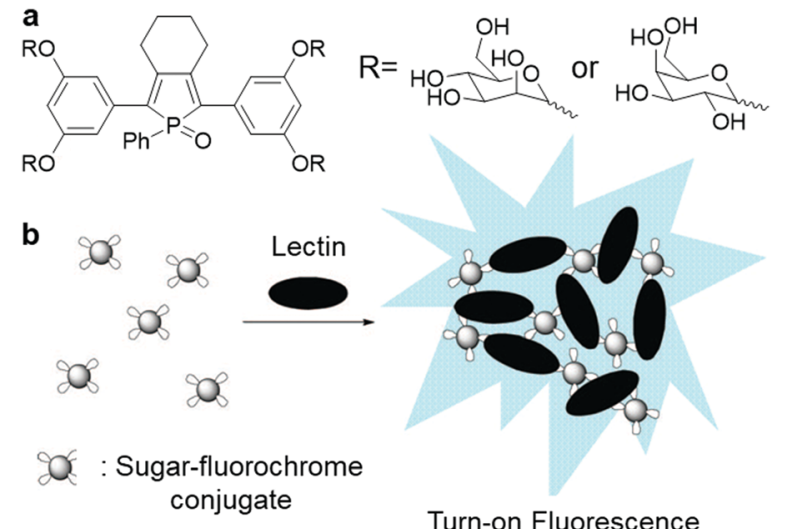

Turn-on Fluorescence

Fig. 5 (a) Chemical structure of sugar-phosphole oxide conjugate and (b) illustration of the operation mechanism for sugar-phosphole oxide conjugate. Adapted and modified with permission from ref. 9. Copyright 2009 American Chemical Society.

bioprobes were designed by the same group to achieve improved sensitivity with a detection limit of up to $20 \mathrm{nM}$ Con A. ${ }^{35}$ Similarly, TPE bioprobes with multivalent mannosyl and cellobiosyl recognition moieties were devised to study lectin and glycosidase activity, respectively. Lately, Hua and He et al. constructed a red to near-infrared emissive AIE-active glycosyl 1,4-diketo-3,6-diphenylpyrrolo[3,4-c]pyrrole (glyco-DPP) probe for the detection of lectin. ${ }^{36}$ Benefitting from its high sensitivity and near-infrared fluorescence response, this probe was only used to accurately quantify lectin in serum samples, but also used for lectin-specific in vivo deep imaging.

Besides cancer-related protein, AIEgen-carbohydrate bioconjugates can also be applied for pathogen detection. Pathogen is a broad term that describes an agent that is infectious, for example bacteria, viruses, fungi and other microorganisms. Although pathogen detection is critical to diagnose infection sources and ensure food safety, sensitive yet rapid sensing strategies are highly demanded since the existing methods such as bacteria plating and gene microarray require painstakingly long diagnosis times and complicated operation procedures. To overcome this obstacle, AIEgen bioconjugates can be applied through prudently-tailored designs to target and detect specific pathogens through binding of the pathogen-associated protein (e.g. toxin). Most of the bacterial enterotoxins are known to preferably bind to oligosaccharide-containing glycosphingolipids on the host cell surface. ${ }^{37-39}$ Accordingly, oligosaccharides have become key molecules in the development of pathogen-targeting AIEgen bioprobes. It is also known that most sugar ligands suffer from weak binding to their protein acceptors, and thus the specificity and affinity of these interactions must rely heavily on the multivalency of the targeting probe. ${ }^{40,41}$

Following the above design strategy, a multi-valent lactosyl (Lac) moiety-bearing TPE-glycoconjugate, Lac-TPE (Scheme 5), was synthesized for the turn-on detection of cholera toxin (CT). ${ }^{42}$ Cholera is an acute intestinal infection triggered by CT-secreted bacterium Vibrio cholerae, which represents a major threat to human, especially in developing countries,

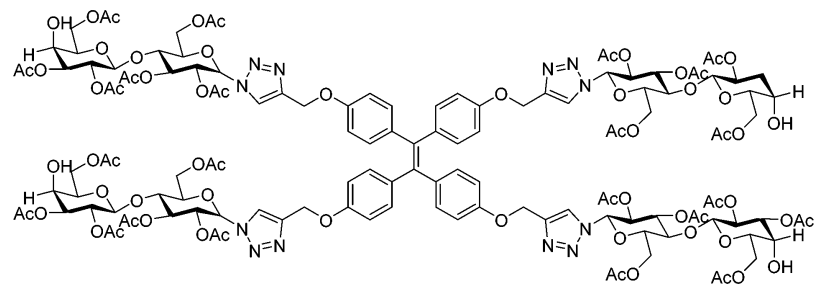

Scheme 5 Chemical structure of Lac-TPE.

where prolonged infection may even cause death due to severe dehydration and electrolyte imbalance. Thus, ultrasensitive probes are in demand to diagnose cholera early, and Lac-TPE is able to fulfil this role. Although Lac-TPE is non-luminescent in blank solution, this probe forms aggregates with cholera toxin B-subunits (CTB) in CTB-presented solution owing to the specific binding of lactose with the cholera toxin B subunit and switches on the fluorescence. The fluorescence spectrum of Lac-TPE showed about 7.5-fold fluorescence intensity enhancement in the presence of $5.0 \mu \mathrm{M}$ CTB. Aside from cholera diagnosis, AIEgen bioconjugates have also been employed to detect influenza. Influenza is one of the most common infectious diseases, which can potentially evolve to be a menace to humanity, as proven by the H3N2 and H1N1 outbreaks in the past few years. With the knowledge of saccharide-protein interaction between saccharide and hemagglutinin, a viral membrane protein on the cell surface, 6 '-sialyllactose moietyconjugated TPE bioprobe, was constructed, which is able to selectively detect human influenza A virus with a concentration higher than $105 \mathrm{pfu} / 100 \mu \mathrm{L} .^{43}$

Another simple bio-conjugated AIE bioprobe, AIE-2Van (Fig. 6a), consisting of a fine-tuned TPE derivative with emission at a wavelength of $650 \mathrm{~nm}$ and vancomycin, a glycopeptide antibiotic, was developed for the specific recognition of Grampositive bacteria, including vancomycin-resistant Enterococcus (VRE) strains. ${ }^{44}$ Fluorescence imaging of bacteria labelled with AIE-2Van in Fig. 6b proved that this bioprobe only exhibited bright red fluorescent emission upon selective binding to Gram-positive bacteria. This is because the conjugated vancomycin has a specific binding affinity towards the $N$-acyl-D-Ala-DAla peptidoglycan on bacterial cell walls, which exists only in

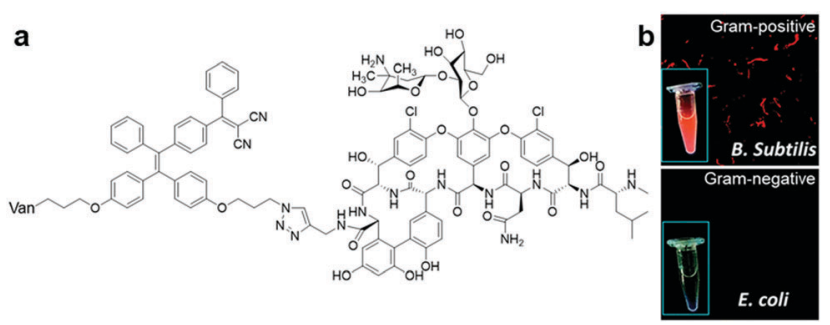

Fig. 6 (a) Chemical structure of AlE-2Van, and (b) fluorescent imaging of Gram-positive (top) and Gram-negative(bottom) bacteria in the presence of $\mathrm{AlE}-2 \operatorname{Van}(0.5 \mu \mathrm{M})$, inset is the photographs of AlE-2Van $(20 \mu \mathrm{M})$ incubated with Gram-positive (top) and Gram-negative(bottom) bacteria under UV irradiation. Adapted and modified with permission from ref. 44. Copyright 2015 Royal Society of Chemistry. 
Gram-positive bacteria. Due to its divalent vancomycin moiety, which can enhance the binding affinity towards Gram-positive bacteria, AIE-2Van can even bind to two VRE strains, Enterococcus faecium and Enterococcus faecalis, to a certain extent. By using a high concentration of AIE-2Van combined with graphene oxide as a background fluorescence quencher, one can observe and differentiate Gram-positive bacteria from Gram-negative ones and VRE strains from unmutated ones under a UV lamp with only the naked eyes (Fig. 6b inset).

\subsection{AIEgen bioconjugate probe based on enzyme cleavage}

Similar to AIEgen bioconjugate probes based on protein-binding and recognition, the operation mechanism of light-up AIEgen bioconjugate probes based on enzyme cleavage is also aimed for RIM activation of the probe, thus inducing fluorescence light-up. The only difference is that while the RIM of AIE bioprobes are activated through binding of the target protein in the first case, the bioprobes in the second case depend on the enzyme cleavage process, which turns the water-soluble probe into a hydrophobic AIEgen residue by specifically cleaving hydrophilic the peptide moiety, and hence results in aggregation of AIEgen and fluorescence turn-on in situ. Based on the site specific cleavage activation mechanism, myriads of AIEgen bioconjugates have been designed for the sensing of enzymes.

3.2.1 AIEgen-peptide bioconjugate. Through conjugation of TPE with the well-known caspase-3 peptide substrate DEVD, the AIEgen-peptide bioconjugate Ac-DEVDK-TPE was reported by Liu and Tang et al. to determine caspase-3/7 activity in realtime based on the site-specific peptide cleavage mechanism (Fig. 7a). ${ }^{10}$ Caspase is a class of intracellular cysteine aspartate protease enzymes in charge of inflammation and cell apoptosis, with caspase-3 playing a pivotal role in the execution of apoptosis. Several critical diseases, for example cancers, neurodegenerative diseases, and atherosclerosis, have been connected to the mis-regulation of cell apoptosis. Hence, real-time tracing of caspase-3 and cell apoptosis with excellent sensitivity is of

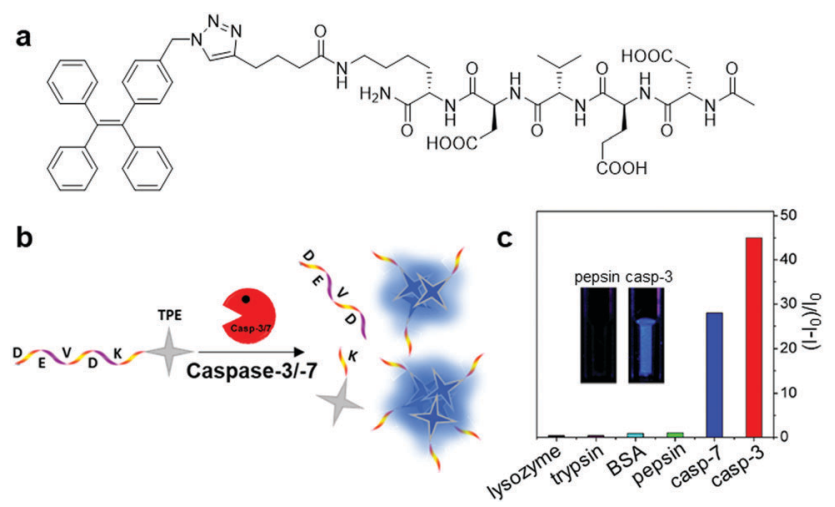

Fig. 7 (a) Chemical structure of Ac-DEVDK-TPE, (b) schematic illustration of the operation mechanism for Ac-DEVDK-TPE, and (c) graph of the fluorescence intensity ratio of Ac-DEVDK-TPE $(10 \mu \mathrm{M})$ versus different proteins (100 pM) with excitation at $312 \mathrm{~nm}$, inset is photographs of Ac-DEVDK-TPE incubated with pepsin and caspase-3, respectively, under UV light. Adapted and modified with permission from ref. 10. Copyright 2014 American Chemical Society. paramount importance for the early diagnosis and progress evaluation of diseases. The operation mechanism of Ac-DEVDK-TPE is based on the site-specific cleavage of the hydrophilic DEVD peptide substrate by caspase-3/7, which releases the hydrophobic AIEgen, and subsequent aggregation of AIEgens will turn on bright blue fluorescence at a wavelength of approximately $470 \mathrm{~nm}$. The AIE probe showed a 75 -fold fluorescence intensity increment upon recognition of caspase-3/-7, and otherwise remained non-fluorescent in aqueous buffer (Fig. 7b). Also, there is clear evidence from the photoluminescence spectra in Fig. 7c that Ac-DEVDK-TPE can only be specifically activated by caspase-3/7. Moreover, this probe enabled live cell apoptosis imaging in realtime, which further provided a new strategy for the in situ screening of apoptosis-associated agents and evaluation of drug efficacy.

While single fluorophore-based AIEgen probe is effective for the assigned tasks in tracing target proteins, fluorescence of the probe could be affected and compromised by autofluorescence from living cells and their microenvironment as well as by errors from drifts of light sources and detectors, therefore results in false and inaccurate conclusions. To overcome this dilemma, ratiometric fluorescent bioprobes with dual fluorophores can be developed, which provide a built-in self-validation mechanism by measuring the fluorescence intensity ratio of two different wavelengths emitted by the probe. A self-validated fluorescence resonance energy transfer (FRET)-based probe, Cou-DEVDTPETH (Fig. 8a), containing a coumarin fluorophore as the donor and TPETH as the acceptor/quencher with a caspase-3 specific DEVD peptide connecting the two fluorophores was reported for the study of biological processes related to caspase- 3 in real time. ${ }^{45}$ Cou-DEVD-TPETH is non-emissive in aqueous solution since the energy is transferred from the donor to the AIEgen-acted quencher via the energy-dissipating RIM mechanism, and only displays strong green (from coumarin) and red (from TPETH) fluorescence simultaneously upon cleavage by caspase-3 due to the separation of coumarin from TPETH as well as subsequent aggregation of the freed AIEgen. Consequently, this dual fluorescence switch-on

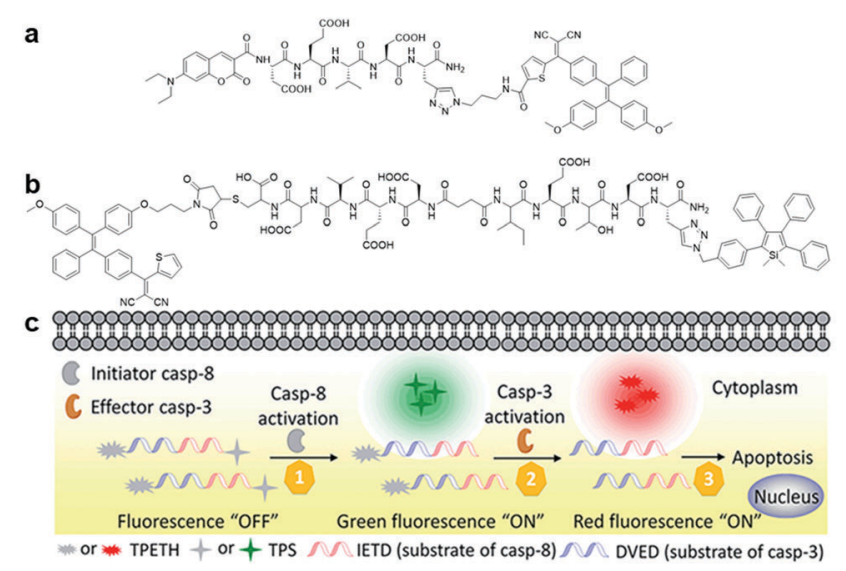

Fig. 8 (a) Chemical structure of Cou-DEVD-TPETH, (b) chemical structure of TPETH-DVEDIETD-TPS, and (c) schematic illustration of caspase- 8 and caspase- 3 sensing by TPETH-DVEDIETD-TPS. Adapted and modified with permission from ref. 46. Copyright 2017 Royal Society of Chemistry. 
probe design enables self-validated detection and real-time monitoring of the target protein with high contrast.

Besides self-validation, dual fluorophore-based AIEgenpeptide conjugate probes can also be utilized for the monitoring of probe interactions with multiple target proteins to study a given biological process through distinct signals. A dual AIEgen bioprobe with a hydrophilic peptide responsive to the apoptosis initiator caspase-8 and effector caspase-3 as a linker, TPETHDVEDIETD-TPS (Fig. 8b), was introduced for the real-time study of early apoptotic events in tumour cells. ${ }^{46}$ The detection of the caspase cascade activation is important for screening potential anticancer drugs and study of drug efficacy since most drugs induce cell death via the apoptosis pathway. During the process of drug-induced apoptosis, the initiator caspases (caspase-8 for example) take charge in activating the effector caspase (such as caspase-3) and subsequently lead to cell apoptosis. When the peptide moiety is cleaved by caspase- 8 and caspase- 3 sequentially in the apoptotic cells, TPETH-DVEDIETD-TPS lights up with intense green (from TPS) and red (from TPETH) fluorescence, respectively, thus enabling multiplexed imaging of caspase activities (Fig. 8c). This probe design paved the way for the multiplexed monitoring of involved proteins in a biological process.

With the proper design of the peptide moiety and assay method, AIEgen bioconjugates can also be tuned to detect deacetylation-catalysing enzymes. Recently, a sirtuin type 1 (SIRT1) modulator screening assay based on an SIRT1 responsive peptide substrate conjugated TPE probe, TPE-GK(Ac)YDD, was constructed (Fig. 9a). ${ }^{47}$ As a member of the evolutionarily conserved intracellular protein deacetylases, SIRT1 is the key nicotinamide adenine dinucleotide (NAD)-dependent enzyme for catalysing deacetylation reactions. Its connection to metabolic disorders and aging-related disease has drawn the attention of scientists, which led to the discovery and development of SIRT1 activators and inhibitors. TPE-GK(Ac)YDD was synthesized by incorporating the lysine-acetylated lysyl endopeptidase responsive peptide substrate, GK(Ac)YDD, to a blue-emissive TPE core via a simple carboxylic acid-amine coupling method. The fluorescence switch-on of TPE-GK(Ac)YDD relied initially on the deacetylation of the acetyl group by SIRT1, followed by

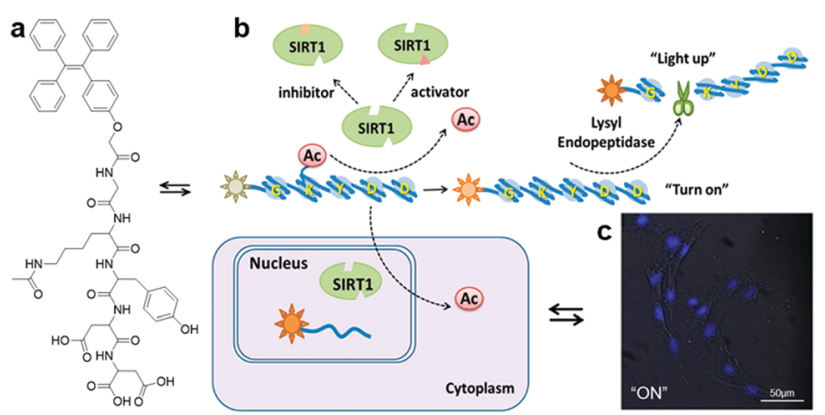

Fig. 9 (a) Chemical structure of TPE-GK(Ac)YDD, (b) scheme of SIRT1 detection by TPE-GK(Ac)YDD, and (c) confocal fluorescence images of SIRT1-overexpressed live cells after incubation with TPE-GK(AC)YDD $(50 \mu \mathrm{M})$. Adapted and modified with permission from ref. 47. Copyright 2015 American Chemical Society. subsequent peptide cleavage by the lysyl endopeptidase introduced additionally, and finally aggregation of the released TPE motifs (Fig. 9b). Thus, this probe performed superbly in the screening of modulators through measuring SIRT1 activity and tracking of endogenous SIRT1 in living cells with strengthened selectivity and sensitivity (Fig. 9c).

Although most of the site-specific cleavage-activated AIEgenpeptide bioconjugates have relied on simple AIEgen molecule aggregation to achieve the fluorescence turn-on state, Liang et al. recently presented a "smart" AIE dimer probe for the visualization of furin overexpressed in cancer cells with magnified fluorescence intensity (Fig. 10a). ${ }^{48}$ This probe was comprised of a TPE unit and furin-targeting 2-cyanobenzothiazole (CBT)-linked $\mathrm{R}(\mathrm{Ac}) \operatorname{VRRC}(\mathrm{StBu}) \mathrm{K}$ peptide. After reduction of the disulfide bond on the cysteine motif by intracellular glutathione and cleavage of RVRR peptide by furin, peptide-cleaved intermediates with freed 1,2-aminothiol group were yielded, which subsequently underwent an intermolecular bio-compatible click condensation reaction between the 1,2-aminothiol group and CBT group to form hydrophobic AIE dimer probes that self-aggregated into AIE nanoparticles (Fig. 10b). Compared to the standard furin-cleavable AIEgen-peptide bioconjugates, the luminescence of this smart AIE dimer probe was amplified by up to 3.4 times for furin activity sensing (Fig. 10c). With these favourable results, one can ambitiously predict that this design strategy will help to create AIEgen bioprobes with further improved detection sensitivity.

In addition to the above-mentioned AIE bioprobes, many other excellent AIEgen bioconjugates based on enzyme cleavage that were not elaborated on in this mini-review are summarized in Table 2 with information about their target enzymes, associated diseases, respective AIEgen bioconjugates for detection of target enzyme and their responsive peptide sequences. ${ }^{49-54}$

3.2.2 AIEgen-carbohydrate bioconjugate. Interestingly, there are several reported AIEgen-carbohydrates that have shown applicability in the sensing of glycoside hydrolase enzyme through

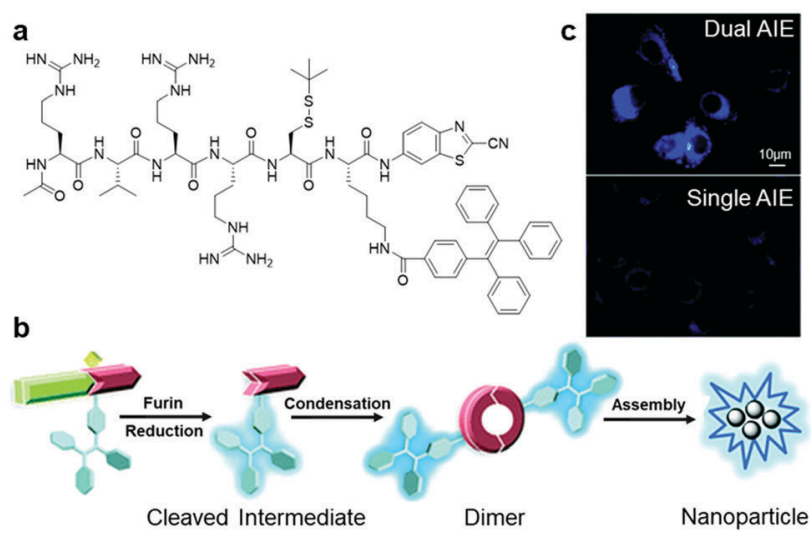

Fig. 10 (a) Chemical structure of Ac-RVRR-Cys(StBu)-Lys(TPE)-CBT, (b) reaction scheme of "smart" AIE dimer and nanoparticle formation upon interacting with furin, and (c) fluorescence imaging of MDA-MB-468 cells incubated with Ac-RVRR-Cys(StBu)-Lys(TPE)-CBT (5 $\mu \mathrm{M})$ (top) and single AlEgen control probe $(5 \mu \mathrm{M})$ (bottom). Adapted and modified with permission from ref. 48. Copyright 2017 Royal Society of Chemistry. 
Table 2 AlEgen biconjugates for specific enzyme sensing

\begin{tabular}{llll}
\hline Target enzyme & Associated disease & AIEgen bioconjugate & Responsive peptide sequence \\
\hline Caspase 1 & Autoinflammatory diseases & TPETH-2(DDYVADC) & YVAD \\
Chymase & Allergic asthma & TPETH-2(CFTERD $\left.)_{3}\right)$ & CFTER \\
Angiotensin converting enzyme & Cardiovascular diseases & TPE-SDKP & SDKP \\
Dipeptidyl peptidase-4 & Type 2 diabetes mellitus & TPE-KFPE & KFPE \\
Matrix metalloproteinase-2 & Cancers & TPE-PLGVR $-\left(\text { PEG }_{8}\right)_{2}$-K(PpIX) & PLGVR \\
& & DOX-FCPPs-TPE-Py & LGLAG
\end{tabular}

the same enzyme cleavage mechanism applied in AIEgen-peptide probes. For instance, an AIE-active salicylaldehyde azine-based probe, SA- $\beta$ Gal (Fig. 11a), possessing $\beta$-galactopyranosides, a D-galactose monosaccharide derivative, was reported to detect $\beta$ - galactosidase, an important biomarker related to ovarian cancer cells and senescent cells. ${ }^{55}$ After interacting with $\beta$-galactosidase, the $\mathrm{D}$-galactose substrate was cleaved to release the green-fluorescent salicylaldehyde azine moiety with both AIE and excited-state intramolecular proton transfer (ESIPT) properties. Due to its unique characteristics, SA- $\beta$ Gal exhibits greatly reduced self-quenching in the aggregate state and large Stokes shift of $190 \mathrm{~nm}$. Hence, SA- $\beta$ Gal can sense the target protein with a detection limit of $0.014 \mathrm{U} \mathrm{mL}^{-1}$ and an impressive light-up ratio of 820 . This bioprobe can also image intracellular $\beta$-galactosidase activity and distinguish $\beta$-galactosidaseoverexpressed cells from others evidently (Fig. 11b). Tang et al. further developed a TPE-based $\beta$-galactosidase responsive probe, TPE-Gal, with a positively charged pyridinium pendant to enhance the water solubility of the probe and D-galactose residue at the probe end as the substrate. ${ }^{56}$ Incubation of this probe with $\beta$-galactosidase resulted in the cleavage of the $\beta$-galactopyranoside group from the probe, generating a phenolate intermediate, which can spontaneously undergo 1,6-elimination of $p$-quinone-methide to yield the TPE moiety with poor hydrophilicity, which then selfaggregated to light up with intense green fluorescence. Compared to SA- $\beta$ Gal, TPE-Gal was able to detect $\beta$-galactosidase at a wider linearity range from 0.8 to $4.8 \mathrm{U} \mathrm{mL}^{-1}$ with a higher detection limit of $0.33 \mathrm{U} \mathrm{mL}^{-1}$.

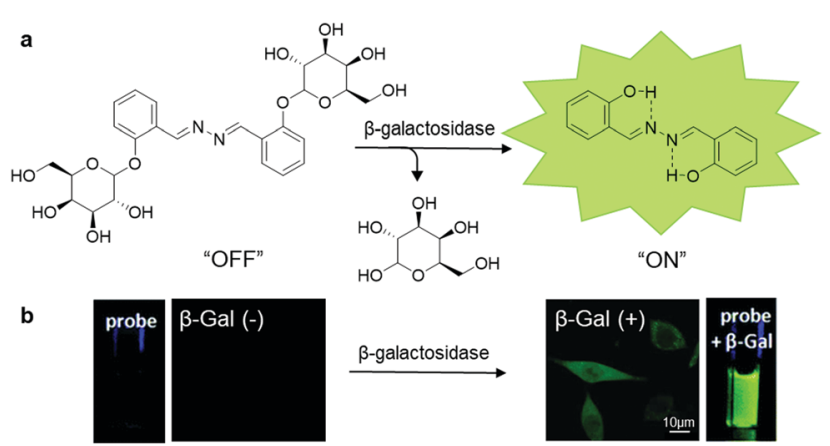

Fig. 11 (a) Chemical structure of $S A-\beta G a l$ and scheme of SA- $\beta$ Gal for $\beta$-galactosidase responsive sensing and imaging, and (b) photographs of SA- $\beta$ Gal $(100 \mu \mathrm{M})$ with (right) and without (left) the presence of $\beta$-galactosidase $\left(4 \mathrm{U} \mathrm{mL} \mathrm{L}^{-1}\right)$ under $\mathrm{UV}$ irradiation, and confocal fluorescence images of $\beta$-galactosidase-positive (right) and $\beta$-galactosidase-negative (left) live cells after incubation with SA- $\beta$ Gal $(50 \mu \mathrm{M})$. Adapted and modified with permission from ref. 55. Copyright 2015 Royal Society of Chemistry.

\subsection{Theranostic AIEgen bioconjugate}

In the past few years, scientists have enthusiastically explored and designed precise targeted therapy systems with built-in real-time in situ reporting of therapeutic processes. Taking the advantage of the sensitive detection of disease-related proteins offered by AIEgen bioconjugates, the fusing of AIEgen bioconjugates and therapeutic modality can yield disease-related protein targeted theranostic platforms, which can provide ultrasensitive detection of target proteins, precise and effective therapy, and report therapeutic responses through protein activity monitoring. ${ }^{57}$

Although chemotherapy serves as the primary therapy for cancer, many patients are traumatized by the side effects associated with chemotherapy due to the indiscriminate attack of chemo drugs towards all cells. Hence, this issue has prompted researchers to search for methods to improve chemotherapy medication for effective, precise and safer cancer treatment. One way to mitigate this challenge is to incorporate a targeting moiety to the chemotherapy medication for targeted drug delivery. To help visualize such targeted delivery and therapeutic response, AIEgen bioconjugates can be tagged onto the prodrug. Based on this design principle, an AIEgenpeptide-prodrug conjugate, TPS-DEVD-Pt-cRGD (Fig. 12a), which is composed of an integrin $\alpha_{v} \beta_{3}$-targeted chemotherapeutic platinum(Iv) prodrug with integrated AIE turn-on apoptosis sensor, was reported in 2014 by Tang and Liu et al. ${ }^{11}$ Upon cRGD-facilitated cellular uptake by integrin-positive cells, the prodrug was reduced to the active $\mathrm{Pt}(\mathrm{II})$ drug and free apoptosis sensor, TPS-DEVD, concurrently. The active Pt(II) drug could then interfere with DNA replication and induce cell apoptosis, and in turn activate caspase-3, which turned on the apoptosis sensor through DEVD peptide cleavage, thus confirming the positive therapeutic response. The mechanism was confirmed by the fluorescence increase in the integrinoverexpressed U87-MG cells upon incubation with the TPSDEVD-Pt-cRGD prodrug compared to the nearly zero emission of the prodrug from integrin-negative MCF7 and 293T cells. Another theranostic prodrug based on gemcitabine (GEM), which is responsive to caspase-3 and cathepsin-B (Fig. 12b), was devised for pancreatic cancer cell-targeted drug delivery and real-time evaluation of therapeutic effects. ${ }^{58}$ This prodrug consisted of the cathepsin-B activatable gemcitabine prodrug, GFLG-GEM, and integrin-targeting apoptotic sensor, TPEDEVD-RGD. After internalization of the prodrug in cathepsinB overexpressed pancreatic tumour cells, active gemcitabine and the apoptotic probe were released simultaneously since the 
a

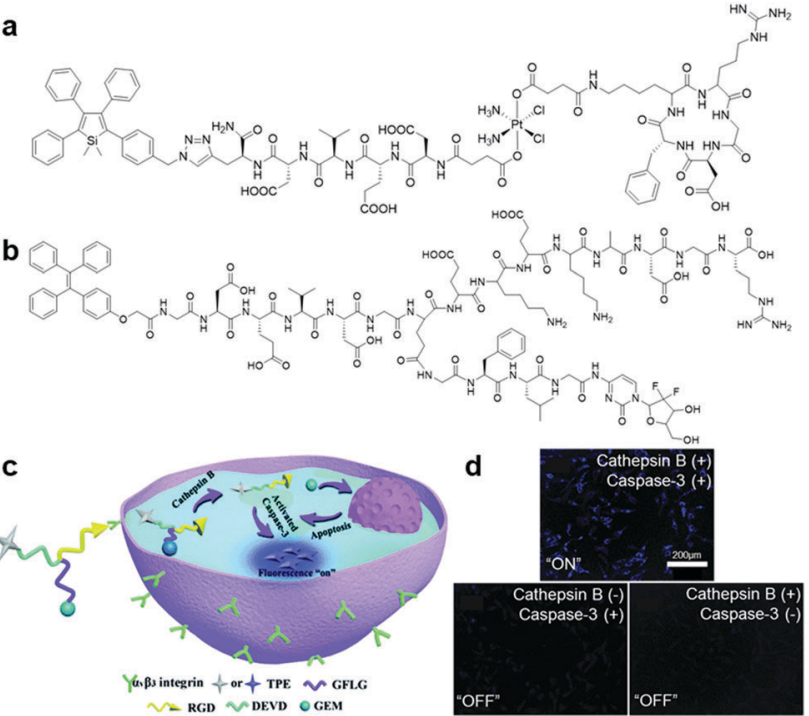

Fig. 12 (a) Chemical structure of TPS-DEVD-Pt-cRGD, (b) chemical structure of TPE-conjugated theranostic GEM prodrug, (c) schematic illustration of TPE-conjugated theranostic GEM prodrug for integrin and cathepsin-B targeted drug delivery and in situ reporting of apoptotic process, and (d) confocal fluorescence images of live cells incubated with the TPE-conjugated theranostic GEM prodrug under different conditions. Adapted and modified with permission from ref. 58. Copyright 2017 Royal Society of Chemistry.

GFLG peptide substrate on the prodrug is specifically cleaved by the cathepsin-B enzyme, which killed the targeted cell and reported the therapeutic efficacy, respectively (Fig. 12c). Upon incubation of this cathepsin-B activatable gemcitabine prodrug, the blue fluorescence signal of the cells overexpressing both integrin and cathepsin-B increased proportionally to the cellular apoptotic progress, while only weak fluorescence emission was observed for both integrin-negative and cathepsin-positive and integrin-positive and cathepsin negative cells (Fig. 12d). Overall, this intracellular cascade enzymatic reaction activatable GEM prodrug has potential to be translated into theranostic medicine for simultaneous pancreatic cancer therapy and real-time noninvasive monitoring of therapeutic responses.

Beyond monitoring of drug delivery and therapeutic response, AIEgen bioconjugates can also play a second role as a therapeutic moiety in the process of disease-related protein detection. In the search for red-emissive AIEgens for probe design, researchers have found that the introduction of $\mathrm{D}-\mathrm{A}$ structure into TPE can also render AIEgens with the ability to produce reactive oxygen species (ROS) upon light irradiation in high efficiency. This ability makes AIEgens competent to serve as photosensitizers (PS) in photodynamic therapy, which is a non-invasive modality for disease such as cancer and infectious inflammation. ${ }^{59}$ With the aid of a targeting moiety, the AIE PS can be delivered to the target efficaciously and execute the command of target eradication once it is activated by light excitation. For example, the aforementioned TPE-red-2AP2H is able to fulfil targeted photodynamic ablation of LATAM4Brelated cancer cells with the guidance of the target ligand AP2H. ${ }^{25}$
More recently, a dual protein-targeted enzyme-activatable bioprobe incorporating an AIE-based photosensitizer, TPECM2GFLGD ${ }_{3}$-cRGD (Fig. 13a), was devised to accomplish concurrent turn-on fluorescence bioimaging and visible light activated photodynamic ablation of targeted cancer cells. ${ }^{60}$ This bioprobe contained four parts: TPECM, an optimized AIE-based PS; tumour-overexpressed cathepsin B-cleavable GFLG peptide; hydrophilic $\mathrm{D}_{3}$ peptide; and integrin $\alpha_{\mathrm{v}} \beta_{3}$-targeting cRGD peptide. TPECM remained inactive in solution due to the strengthened solubility offered by the conjugated hydrophilic peptide. Upon internalization of TPECM facilitated by the cRGD peptide moiety and subsequent cleavage of the GFLG peptide substrate by intracellular cathepsin-B enzyme, this AIE-based PS immediately aggregated and was activated to carry out its role as a theranostic agent (Fig. 13b). The excellent target specificity of this probe was proven through extensive cellular experiments, where the probe only gives intense fluorescence in cells with both active integrin $\alpha_{v} \beta_{3}$ and cathepsin B (Fig. 13c). As a result, after light-triggered photodynamic therapy, this probe only caused grave cell ablation to integrin $\alpha_{v} \beta_{3}$ and cathepsin B overexpressed MDA-MB-231 cells over the other two negative controls, MCF-7 and 293T cells. Another bioprobe, TPETH-SS-DEVD-TPS-cRGD (Fig. 13d), was designed for targeted photodynamic cancer therapy and real-time monitoring of probe response in tumour cells. ${ }^{61}$ Upon specific uptake by integrin $\alpha_{v} \beta_{3}$ overexpressed cancer cells and subsequent intracellular disulfide linkage reduction, red-fluorescent TPE-based PS is separated from the apoptosis sensor to report successful probe internalization and initiation of photodynamic cell ablation. Activated caspase-3/-7 during cell apoptosis induced by generated reactive oxygen species then turns on the apoptosis sensor via DEVD peptide substrate cleavage to afford bright green fluorescence. Most importantly, the fluorescence turn-on of both AIEgens can easily be achieved with single

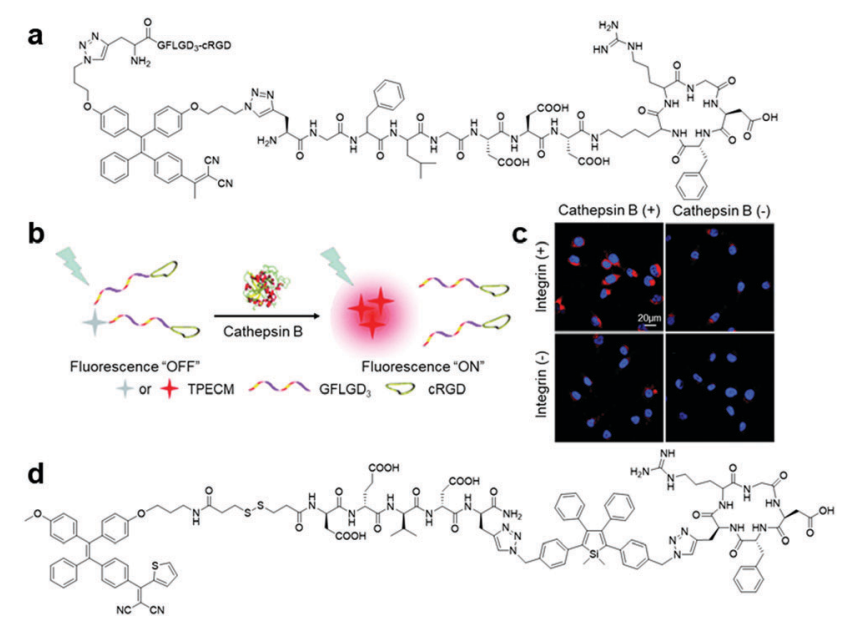

Fig. 13 (a) Chemical structure of TPECM-2GFLGD 3 -CRGD, (b) schematic illustration of the operation mechanism for TPECM-2GFLGD 3 -CRGD, (c) confocal fluorescence images of live cells after incubation with TPECM-2GFLGD 3 -CRGD $(5 \mu \mathrm{M})$ under different conditions, and (d) chemical structure of TPETH-SS-DEVD-TPS-CRGD. Adapted and modified with permission from ref. 60. Copyright 2015 Wiley-VCH. 


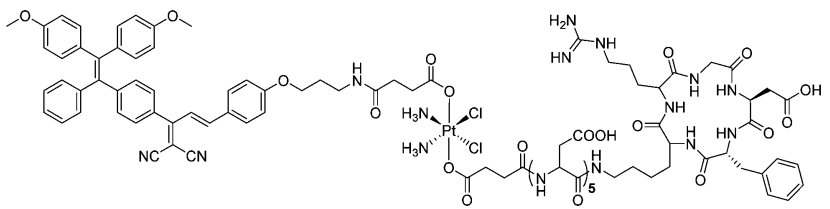

Scheme 6 Chemical structure of TPECB-Pt-D5-cRGD

wavelength excitation, hence the whole therapeutic response in a single cell can be captured in detail without effort.

With of the increase in drug resistant cancer cells as a great concern, dual or multi-modality-based combinational therapy has recently been considered as a promising technique to increase cell ablation efficiency. In one of the recent examples demonstrating this effort, an $\alpha_{\mathrm{v}} \beta_{3}$ integrin targeted platinum(Iv) prodrug conjugated with an AIE-based PS, TPECB-Pt-D5-cRGD (Scheme 6), was introduced for monitoring and combinational therapy of integrin overexpressed cisplatin-resistant cancer cell. $^{62}$ This prodrug can be activated by intracellular glutathione and release the bonded AIE PS upon internalization into targeted cells. The AIE PS will then form aggregates, which can be used as a fluorescent reporter to confirm successful cell internalization and drug activation as well as photodynamic therapy via light irradiation induced ROS generation. To validate the performance of the designed TPECB-Pt-D5-cRGD, the probe was incubated with integrin overexpressed cisplatin-resistant MDA-MB-231 cells. Fascinatingly, not only fluorescence turn-on was observed only in MDA-MB-231 cells 4 hours after the incubation under confocal microscopy imaging with MCF-7 cells as a negative control, the half-maximum inhibitory concentration $\left(\mathrm{IC}_{50}\right)$ of TPECB-Pt-D5-cRGD was also found to decrease from $37.1 \mu \mathrm{M}$ under dark conditions to $4.2 \mu \mathrm{M}$ upon light irradiation during the testing of anti-proliferative properties, thus illustrating the targeting specificity and high combinational therapy efficacy of the probe.

\section{Conclusion and outlook}

Due to their unique aggregation-induced emission characteristics, AIEgens are becoming more and more prominent among the fluorogenic molecules and have successfully attracted attention from researchers because of their boundless potential in various biological applications. In this mini-review, the design and synthetic strategies of AIEgen bioconjugates were presented, and examples of AIE-based bioprobes for protein and pathogen sensing in recent years were discussed. From these examples, one can observe that through ingenious design, bioconjugated AIE probes are able to sense and respond to a variety of proteins and pathogens with high sensitivity and selectivity. Aside from the typical RIM-induced AIE effect, additional synergistic mechanisms for enhanced SNR in target detection have also been introduced, such as fluorescence output enhancement induced by self-assembly aided peptides, "smart" AIE dimer fluorescence enhancement, dual fluorogen-based ratiometric comparison, FRET and ESIPT, which further broaden the feasible probe design. Moreover, bioprobes can even be tailored to be multifunctional by integrating multiple recognition elements and prodrugs into a single system, thus enabling multiplexed sensing of target proteins, image-guided precise drug delivery, dual modalities based combinational therapy, and real-time monitoring of therapeutic effects.

It is encouraging that progress and milestones have been achieved in the development of AIEgen bioconjugates for protein and pathogen sensing throughout the years; however, there are still many crucial aspects to be considered for the further advancement of AIEgen-based bioprobes. Although the ultimate goals of these reported bioprobes are to be utilized for in vivo clinical studies, the incorporation of TPS or TPE fluorogens has limited the smooth transition to practical applications due to their short absorption wavelength, poor light penetrating depth and emission overlap with autofluorescence. Thus, one course of action is to develop new far-red or near-infrared emissive AIEgens with long-wavelength absorption. Alternatively, one can design AIEgens with large multiphoton absorption cross-sections for precise multiphoton imaging. Also, the development of AIE luminogens with room-temperature phosphorescence will also help in eliminating autofluorescence and simultaneously enable long-term tracking of target analytes. Additionally, although peptides were preferred as recognition elements for most of the reported AIEgen biosensors, aptamers have started to emerge in the design of probes, which need further exploration. Lastly, other stimuli-responsive moieties such as mechanical or thermalactivatable molecules can be introduced into AIEgen bioconjugates as well to boost the controllability of the probes in various applications.

\section{Conflicts of interest}

There are no conflicts to declare.

\section{Acknowledgements}

The authors are grateful to Singapore National Research Foundation (R279-000-444-281, R279-000-483-281), and National University of Singapore (R279-000-482-133) for financial support.

\section{Notes and references}

1 T. Förster and K. Kasper, Z. Phys. Chem., 1954, 1, 275-277. 2 J. Luo, Z. Xie, J. W. Lam, L. Cheng, H. Chen, C. Qiu, H. S. Kwok, X. Zhan, Y. Liu and D. Zhu, Chem. Commun., 2001, 1740-1741.

3 K. Blennow, NeuroRx, 2004, 1, 213-225.

4 C. A. Borrebaeck, Nat. Rev. Cancer, 2017, 17, 199.

5 S. M. Hewitt, J. Dear and R. A. Star, J. Am. Soc. Nephrol., 2004, 15, 1677-1689.

6 M. Wang, G. Zhang, D. Zhang, D. Zhu and B. Z. Tang, J. Mater. Chem., 2010, 20, 1858-1867.

7 H. Shi, J. Liu, J. Geng, B. Z. Tang and B. Liu, J. Am. Chem. Soc., 2012, 134, 9569-9572.

8 Y. Li, R. T. Kwok, B. Z. Tang and B. Liu, RSC Adv., 2013, 3, 10135-10138. 
9 T. Sanji, K. Shiraishi and M. Tanaka, ACS Appl. Mater. Interfaces, 2008, 1, 270-273.

10 H. Shi, R. T. Kwok, J. Liu, B. Xing, B. Z. Tang and B. Liu, J. Am. Chem. Soc., 2012, 134, 17972-17981.

11 Y. Yuan, R. T. Kwok, B. Z. Tang and B. Liu, J. Am. Chem. Soc., 2014, 136, 2546-2554.

12 Y. Hong, J. W. Lam and B. Z. Tang, Chem. Soc. Rev., 2011, 40, 5361-5388.

13 D. Ding, K. Li, B. Liu and B. Z. Tang, Acc. Chem. Res., 2013, 46, 2441-2453.

14 J. Mei, Y. Hong, J. W. Lam, A. Qin, Y. Tang and B. Z. Tang, Adv. Mater., 2014, 26, 5429-5479.

15 R. Hu, N. L. Leung and B. Z. Tang, Chem. Soc. Rev., 2014, 43, 4494-4562.

16 J. Liang, B. Z. Tang and B. Liu, Chem. Soc. Rev., 2015, 44, 2798-2811.

17 J. Mei, N. L. Leung, R. T. Kwok, J. W. Lam and B. Z. Tang, Chem. Rev., 2015, 115, 11718-11940.

18 R. T. Kwok, C. W. Leung, J. W. Lam and B. Z. Tang, Chem. Soc. Rev., 2015, 44, 4228-4238.

19 G. Feng, R. T. Kwok, B. Z. Tang and B. Liu, Appl. Phys. Rev., 2017, 4, 021307.

$20 \mathrm{~J}$. Mei, Y. Huang and H. Tian, ACS Appl. Mater. Interfaces, 2018, 10, 12217-12261.

21 J. E. Kwon and S. Y. Park, Adv. Mater., 2011, 23, 3615-3642.

22 Y. Huang, F. Hu, R. Zhao, G. Zhang, H. Yang and D. Zhang, Chem. - Eur. J., 2014, 20, 158-164.

23 J. Geng, W. L. Goh, C. Zhang, D. P. Lane, B. Liu, F. Ghadessy and Y. N. Tan, J. Mater. Chem. B, 2015, 3, 5933-5937.

24 N. Pradhan, D. Jana, B. K. Ghorai and N. R. Jana, ACS Appl. Mater. Interfaces, 2015, 7, 25813-25820.

25 F. Hu, Y. Huang, G. Zhang, R. Zhao, H. Yang and D. Zhang, Anal. Chem., 2014, 86, 7987-7995.

26 R. Zhang, G. Feng, C.-J. Zhang, X. Cai, X. Cheng and B. Liu, Anal. Chem., 2016, 88, 4841-4848.

27 R. Zhang, S. H. Sung, G. Feng, C.-J. Zhang, B. Z. Tang and B. Liu, Anal. Chem., 2017, 90, 1154-1160.

28 C. Chen, Z. Song, X. Zheng, Z. He, B. Liu, X. Huang, D. Kong, D. Ding and B. Z. Tang, Chem. Sci., 2017, 8, 2191-2198.

29 Y. Cheng, C. Sun, X. Ou, B. Liu, X. Lou and F. Xia, Chem. Sci., 2017, 8, 4571-4578.

30 R. Zhang, R. T. Kwok, B. Z. Tang and B. Liu, RSC Adv., 2015, 5, 28332-28337.

31 X. Min, Y. Zhuang, Z. Zhang, Y. Jia, A. Hakeem, F. Zheng, Y. Cheng, B. Z. Tang, X. Lou and F. Xia, ACS Appl. Mater. Interfaces, 2015, 7, 16813-16818.

32 J. Chen, H. Jiang, H. Zhou, Z. Hu, N. Niu, S. A. Shahzad and C. Yu, Chem. Commun., 2017, 53, 2398-2401.

33 P. Mallikaratchy, Z. Tang, S. Kwame, L. Meng, D. Shangguan and W. Tan, Mol. Cell. Proteomics, 2007, 6, 2230-2238.

34 K. Ma, F. Zhang, N. Sayyadi, W. Chen, A. Anwer, A. Care, B. Xu, W. Tian, E. M. Goldys and G. Liu, ACS Sens., 2018, 3, 320-326.

35 T. Sanji, K. Shiraishi, M. Nakamura and M. Tanaka, Chem. Asian J., 2010, 5, 817-824.

36 Y. Hang, X.-P. He, L. Yang and J. Hua, Biosens. Bioelectron., 2015, 65, 420-426.
37 C. R. MacKenzie, T. Hirama, K. K. Lee, E. Altman and N. M. Young, J. Biol. Chem., 1997, 272, 5533-5538.

38 A. Aman, S. Fraser, E. Merritt, C. Rodigherio, M. Kenny, M. Ahn, W. Hol, N. Williams, W. Lencer and T. Hirst, Proc. Natl. Acad. Sci. U. S. A., 2001, 98, 8536-8541.

39 H. Nakajima, N. Kiyokawa, Y. U. Katagiri, T. Taguchi, T. Suzuki, T. Sekino, K. Mimori, T. Ebata, M. Saito and H. Nakao, J. Biol. Chem., 2001, 276, 42915-42922.

40 Y. C. Lee and R. T. Lee, Acc. Chem. Res., 1995, 28, 321-327. 41 J. J. Lundquist and E. J. Toone, Chem. Rev., 2002, 102, 555-578.

42 X. M. Hu, Q. Chen, J. X. Wang, Q. Y. Cheng, C. G. Yan, J. Cao, Y. J. He and B. H. Han, Chem. - Asian J., 2011, 6, 2376-2381.

43 T. Kato, A. Kawaguchi, K. Nagata and K. Hatanaka, Biochem. Biophys. Res. Commun., 2010, 394, 200-204.

44 G. Feng, Y. Yuan, H. Fang, R. Zhang, B. Xing, G. Zhang, D. Zhang and B. Liu, Chem. Commun., 2015, 51, 12490-12493.

45 Y. Yuan, R. Zhang, X. Cheng, S. Xu and B. Liu, Chem. Sci., 2016, 7, 4245-4250.

46 Y. Yuan, C.-J. Zhang, R. T. Kwok, D. Mao, B. Z. Tang and B. Liu, Chem. Sci., 2017, 8, 2723-2728.

47 Y. Wang, Y. Chen, H. Wang, Y. Cheng and X. Zhao, Anal. Chem., 2015, 87, 5046-5049.

48 X. Liu and G. Liang, Chem. Commun., 2017, 53, 1037-1040.

49 H. Lin, H. Yang, S. Huang, F. Wang, D.-M. Wang, B. Liu, Y.-D. Tang and C.-J. Zhang, ACS Appl. Mater. Interfaces, 2018, 10, 12173-12180.

50 R. Zhang, C.-J. Zhang, G. Feng, F. Hu, J. Wang and B. Liu, Anal. Chem., 2016, 88, 9111-9117.

51 H. Wang, Y. Huang, X. Zhao, W. Gong, Y. Wang and Y. Cheng, Chem. Commun., 2014, 50, 15075-15078.

52 Y. Wang, X. Wu, Y. Cheng and X. Zhao, Chem. Commun., 2016, 52, 3478-3481.

53 K. Han, S.-B. Wang, Q. Lei, J.-Y. Zhu and X.-Z. Zhang, ACS Nano, 2015, 9, 10268-10277.

54 Y. Cheng, F. Huang, X. Min, P. Gao, T. Zhang, X. Li, B. Liu, Y. Hong, X. Lou and F. Xia, Anal. Chem., 2016, 88, 8913-8919.

55 L. Peng, M. Gao, X. Cai, R. Zhang, K. Li, G. Feng, A. Tong and B. Liu, J. Mater. Chem. B, 2015, 3, 9168-9172.

56 G. Jiang, G. Zeng, W. Zhu, Y. Li, X. Dong, G. Zhang, X. Fan, J. Wang, Y. Wu and B. Z. Tang, Chem. Commun., 2017, 53, 4505-4508.

57 G. Feng and B. Liu, Small, 2016, 12, 6528-6535.

58 H. Han, W. Teng, T. Chen, J. Zhao, Q. Jin, Z. Qin and J. Ji, Chem. Commun., 2017, 53, 9214-9217.

59 J. Qian and B. Z. Tang, Chem, 2017, 3, 56-91.

60 Y. Yuan, C. J. Zhang, M. Gao, R. Zhang, B. Z. Tang and B. Liu, Angew. Chem., Int. Ed., 2015, 54, 1780-1786.

61 Y. Yuan, C. J. Zhang, R. T. Kwok, S. Xu, R. Zhang, J. Wu, B. Z. Tang and B. Liu, Adv. Funct. Mater., 2015, 25, 6586-6595.

62 Y. Yuan, C.-J. Zhang and B. Liu, Chem. Commun., 2015, 51, 8626-8629. 